\title{
Innovative Planning in-Service Inspections of Fatigued Structures Under Parametric Uncertainty of Lifetime Models
}

\author{
Nicholas A. Nechval ${ }^{1, ~ *}$, Vadims Danovics², Natalija Ribakova ${ }^{2}$ \\ ${ }^{1}$ Department of Mathematics, Baltic International Academy, Riga, Latvia \\ ${ }^{2}$ Department of Marketing, University of Latvia, Riga, Latvia \\ Email address: \\ nechval@junik.lv (N. A. Nechval), vadims.danovics@lu.lv (V. Danovics), napasha@inbox.lv (N. Ribakova)
}

\section{To cite this article:}

Nicholas A. Nechval, Vadims Danovics, Natalija Ribakova. Innovative Planning in-Service Inspections of Fatigued Structures Under Parametric Uncertainty of Lifetime Models. American Journal of Theoretical and Applied Statistics. Special Issue: Novel Ideas for Efficient Optimization of Statistical Decisions and Predictive Inferences under Parametric Uncertainty of Underlying Models with Applications. Vol. 5, No. 2-1, 2016, pp. 29-39. doi: 10.11648/j.ajtas.s.2016050201.15

\begin{abstract}
The main aim of this paper is to present more accurate stochastic fatigue models for solving the fatigue reliability problems, which are attractively simple and easy to apply in practice for situations where it is difficult to quantify the costs associated with inspections and undetected cracks. From an engineering standpoint the fatigue life of a structure consists of two periods: (i) crack initiation period, which starts with the first load cycle and ends when a technically detectable crack is presented, and (ii) crack propagation period, which starts with a technically detectable crack and ends when the remaining cross section can no longer withstand the loads applied and fails statically. Periodic inspections of fatigued structures, which are common practice in order to maintain their reliability above a desired minimum level, are based on the conditional reliability of the fatigued structure. During the period of crack initiation, when the parameters of the underlying lifetime distributions are not assumed to be known, for effective in-service inspection planning (with decreasing intervals as alternative to constant intervals often used in practice for convenience in operation), the pivotal quantity averaging (PQA) approach is offered. During the period of crack propagation (when the damage tolerance situation is used), the approach, based on an innovative crack growth equation, to in-service inspection planning (with decreasing intervals between sequential inspections) is proposed to construct more accurate reliability-based inspection strategy in this case. To illustrate the suggested approaches, the numerical examples are given.
\end{abstract}

Keywords: Fatigued Structure, Crack, Initiation, Propagation, In-Service Inspection Planning, Innovative Approaches

\section{Introduction}

Certain fatigued structures must be inspected in order to detect fatigue damages that would otherwise not be apparent. For fatigued structures for which fatigue damages are only detected at the time of inspection, it is important to be able to determine the optimal times of inspections. Fewer inspections will lead to lower fatigue reliability of the structure upon demand, and frequent inspection will lead to higher cost. In spite of decades of investigation, fatigue response of materials is yet to be fully understood. This is partially due to the complexity of loading at which two or more loading axes fluctuate with time. Examples of structures experiencing such complex loadings are automobile, aircraft, off-shores, railways and nuclear plants. Fluctuations of stress and/or strains are difficult to avoid in many practical engineering situations and are very important in design against fatigue failure. While most industrial failures involve fatigue, the assessment of the fatigue reliability of industrial components being subjected to various dynamic loading situations is one of the most difficult engineering problems. The traditional analytical method of engineering fracture mechanics (EFM) usually assumes that crack size, stress level, material property and crack growth rate, etc. are all deterministic values which will lead to conservative or very conservative outcomes. According to many experimental results and field data, even in well-controlled laboratory conditions, crack growth results usually show a considerable statistical variability. Fatigue is one of the most important problems of aircraft arising from their nature as multiple-component structures, subjected to random dynamic loads. The analysis of fatigue crack growth is one of the most important tasks in the design and life prediction of aircraft fatigue-sensitive structures (for instance, wing, fuselage) and their components (for instance, aileron or balancing flap as part of the wing panel, stringer, etc.). From an engineering standpoint the fatigue life of a structure (or 
component) consists of two periods (this concept is shown schematically in Figure 1):

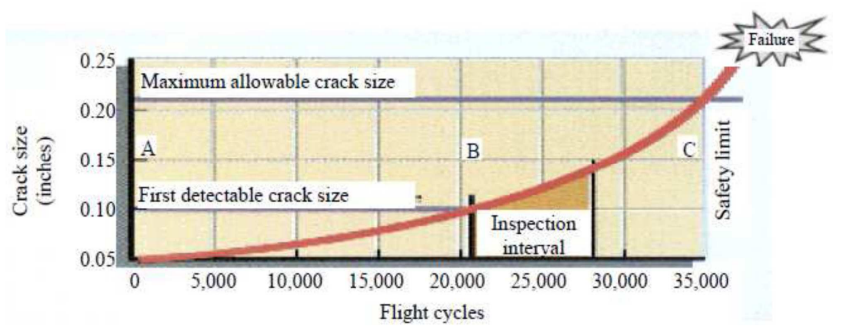

Figure 1. Schematic fatigue crack growth curve (Crack initiation period $(A-B)$; Crack propagation period $(B-C)$ ).

(i) crack initiation period, which starts with the first load cycle and ends when a technically detectable crack is present, and (ii) crack propagation period, which starts with a technically detectable crack and ends when the remaining cross section can no longer withstand the loads applied and fails statically. Periodic inspections of aircraft are common practice in order to maintain their reliability above a desired minimum level.

For guaranteeing safety, the structural life ceiling limits of the fleet aircraft are defined from three distinct situations: Safe-Life, Damage Tolerance, and Fail-Safe situations. The common objectives to define fleet aircraft lives by the three situations are to ensure safety while at the same time reducing total ownership costs. The Safe-Life situation is based on the concept that significant damage, i. e. fatigue cracking, will not develop during the service life of a structure (or component). The life is initially determined from fatigue test data $(S-N$ curves) and calculations using a cumulative damage "law". Then the design Safe-Life is obtained by applying a safety factor. When the service life equals the design Safe-Life the component must be replaced. However, there are two major drawbacks to this situation: (1) components are taken out of service even though they may have substantial remaining lives; (2) despite all precautions, cracks sometimes occur prematurely. This latter fact led airlines to introduce the Damage Tolerance situation (Iyyer et al. [1]). The Damage Tolerance situation recognizes that damage can occur and develop during the service life of a structure (or component). Also, it assumes that cracks or flaws can be present in new structures. Safety is obtained from this situation by the requirements that either (1) any damage will be detected by routine inspection before it results in a dangerous reduction of the static strength (inspectable components), or (2) initial damage shall not grow to a dangerous size during the service life (non-inspectable components). For Damage Tolerance analysis to be successful it must be possible to: (i) Define either a minimum crack length that will not go undetected during routine inspections, or else an initial crack length, nominally based on pre-service inspection capability; (ii) Predict crack growth during the time until the next inspection or until the design service life is reached. The Fail-Safe situation assumes initial damage as manufactured and its subsequent growth during service to detectable crack sizes or greater. Service life in fail-safe structures can thus be defined as the time to a service detectable damage. Inspection intervals are determined by using appropriate safety factors on calculated crack growth time interval from service detectable cracks to critical crack sizes. The prediction of crack growth is similar to that for Damage Tolerance situation, except that a much smaller initial crack length is used.

Many important fatigued structures (for instance, Transportation Systems and Vehicles: aircraft, space vehicles, trains, ships; Civil Structures: bridges, dams, tunnels; and so on) for which extremely high reliability is required are maintained by in-service inspections to prevent the reliability degradation due to fatigue damage. However, temporal transition of the reliability is significantly affected by the inspection strategy selected. Thus, to keep structures reliable against fatigue damage by inspections, it is clearly important in engineering to examine the optimal inspection strategy. In particular, it should be noticed that periodical inspections with predetermined constant intervals are not always effective, since a fatigue crack growth rate is gradually accelerated as fatigue damage grows, i. e. the intervals between inspections should be gradually smaller in order to restrain the reliability degradation by repeated inspections. Therefore, we need to construct the inspection strategy by paying attention to this case. Barlow et al. [2] tackled this problem by assuming a known, fixed cost of making an inspection and a known fixed cost per unit time due to undetected failure. They then found a sequence of inspection times for which the expected cost is a minimum. Their results have been extended by various authors (Luss and Kander [3]; Sengupta [4]). Unfortunately, it is difficult to compute optimal checking procedures numerically, because the computations are repeated until the procedures are determined to the required degree by changing the first check time. To avoid this, Munford and Shahani [5] suggested a sub-optimal (or nearly optimal) but computationally easier inspection policy. This policy was used for Weibull and gamma failure distribution cases (Munford and Shahani [6]; Tadikamalla [7]). Numerical comparisons among certain inspection policies are given by Munford [8] for the case of Weibull failure times. This case under parametric uncertainty was considered by Nechval et al. [9-11].

Most models, which are used for solving the problems of inspection planning, are developed under the assumptions that the parameter values of the models are known with certainty. When these models are applied to solve real-world problems, the parameters are estimated and then treated as if they were the true values. The risk associated with using estimates rather than the true parameters is called estimation risk and is often ignored. In this paper, the case is considered when the functional form of the underlying invariant lifetime distribution is assumed to be known, but some or all of its parameters are unspecified. Periodic inspections of aircraft, which are common practice in order to maintain their reliability above a desired minimum level, are based on the conditional reliability of the fatigued structure. During the period of crack initiation, when the parameters of the 
underlying lifetime distributions are not assumed to be known, for efficient in-service inspection planning (with decreasing intervals as alternative to constant intervals often used in practice for convenience in operation), the pivotal quantity averaging (PQA) approach is offered. During the period of crack propagation (when the damage tolerance situation is used), the approach based on a novel crack growth equation to efficient in-service inspection planning (with decreasing intervals between sequential inspections) is proposed to construct more accurate reliability-based inspection strategy in this case.

\section{Planning in-Service Inspections Under Fatigue Crack Initiation in the Case of Complete Information}

In this paper we look at inspection strategies for items or structures that can be described as being in one of two states, one of which is preferable to the other. This preferred state might be described as 'working' whilst the other may represent some sort of 'fatigue damage'. The structures are originally known to be in a working state but may subsequently have fatigue damage. In other words, at $t_{0}=0$ the structure is in state $S\left(t_{0}\right)$ (working) but at a later time, $t_{1}$, the structure will move into state $S\left(t_{1}\right)$ (fatigue damage). We suppose that we do not know when the transition from $S\left(t_{0}\right)$ into $S\left(t_{1}\right)$ will occur, and that fatigue damage (crack) can only be detected through inspection. We deal with situations, where it is difficult to quantify the costs associated with inspections and undetected fatigue damage, or when these costs vary in time.

\subsection{Inspection Strategy}

The inspection strategy defined is based on the conditional reliability of the structure. It is given as follows. Fix $0<\gamma<1$ and let

$$
\begin{gathered}
\tau_{1}=\arg \left(\operatorname{Pr}\left\{X>\tau_{1}\right\}=\gamma\right), \\
\tau_{j}=\arg \left(\operatorname{Pr}\left\{X>\tau_{j} \mid X>\tau_{j-1}\right\}=\gamma\right), \quad j \geq 2,
\end{gathered}
$$

where $\left\{\tau_{j}\right\}_{j=1,2, \ldots}$ are inspection times, $X$ is a random variable representing the lifetime of the component (structure). This is named as 'reliability-based inspection'. The above inspection strategy makes use of the information about the remaining life that is inherent in the sequence of previous inspection times. The value of $\gamma$ can be seen as 'minimum fatigue reliability required' (or 'fatigue reliability index') during the next period when the structure was still operational at last inspection time, that is, in other words, the conditional probability that the failure (fatigue crack) occurs in the time interval $\left(\tau_{j-1}, \tau_{j}\right)$ without failure at time $\tau_{j-1}$ is always assumed $1-\gamma$. It is clear that if $F_{\theta}$, the structure lifetime distribution with the parameter $\theta$ (in general, vector), is continuous and strictly increasing, the definition of the inspection strategy is equivalent to

$$
\tau_{j}=\arg \left(\bar{F}_{\theta}\left(\tau_{j}\right)=\gamma^{j}\right), \quad j \geq 1
$$

or equivalent to

$$
\tau_{j}=\arg \min _{\tau_{j}}\left[\bar{F}_{\theta}\left(\tau_{j}\right)-\gamma^{j}\right]^{2}, \quad j \geq 1,
$$

where

$$
\bar{F}_{\theta}\left(\tau_{j}\right)=1-F_{\theta}\left(\tau_{j}\right)
$$

\subsection{Optimization of Fatigue Reliability Index}

If it is known that each inspection costs $c_{1}$ and the cost of leaving an undetected failure (fatigue crack) is $c_{2}$ per unit time, then the total expected cost per inspection cycle is given by

$$
\begin{gathered}
C(\tau(\theta, \gamma))=\sum_{j=1}^{\infty} \int_{j-1}^{\tau_{j}}\left[j c_{1}+c_{2}\left(\tau_{j}-x\right)\right] f_{\theta}(x) d x \\
=c_{1} \sum_{j=1}^{\infty} j\left[F_{\theta}\left(\tau_{j}\right)-F_{\theta}\left(\tau_{j-1}\right)\right]+c_{2} \sum_{j=1}^{\infty} \tau_{j}\left[F_{\theta}\left(\tau_{j}\right)-F_{\theta}\left(\tau_{j-1}\right)\right] \\
=c_{1} \sum_{j=0}^{\infty} \bar{F}_{\theta}\left(\tau_{j}\right)+c_{2} \sum_{j=1}^{\infty} \tau_{j}\left[\bar{F}_{\theta}\left(\tau_{j-1}\right)-\bar{F}_{\theta}\left(\tau_{j}\right)\right]-c_{2} E_{\theta}\{X\} \\
=c_{1} \sum_{j=0}^{\infty} \gamma^{j}+c_{2}(x) d x \\
=\frac{c_{1}}{1-\gamma}+c_{2} \varphi(\theta) j \ln \gamma\left[\gamma^{j-1}-\gamma^{j}\right]-c_{2} E_{\theta}\{X\} \\
=\frac{c_{1}}{1-\gamma}+c_{2} \varphi(\theta) \ln \gamma\left(\frac{1-\gamma}{\gamma}\right) \sum_{j=1}^{\infty} j \gamma^{j}-c_{2} E_{\theta}\{X\} \\
=\frac{c_{1}}{1-\gamma}+\frac{c_{2} \varphi(\theta) \ln \gamma}{1-\gamma}-c_{2} E_{\theta}\{X\}, \\
(1-\gamma)^{2} E_{\theta}\{X\}
\end{gathered}
$$

where $f_{\theta}(x)$ is the probability density function of the structure lifetime $X$,

$$
\begin{gathered}
E_{\theta}\{X\}=\int_{0}^{\infty} x f_{\theta}(x) d x, \\
\tau_{j}=\arg \left(\ln F_{\theta}\left(\tau_{j}\right)=\ln \gamma^{j}\right)=\varphi(\theta) j \ln \gamma .
\end{gathered}
$$

Thus, we can choose the fatigue reliability index $\gamma$ such that $C(\tau(\theta, \gamma))$ as defined in (6) is minimized. The appropriate value of $\gamma$ is determined from $\partial C(\tau(\theta, \gamma))\} / \partial \gamma=0$, and we get the optimal value of the fatigue reliability index as 


$$
\gamma^{*}=\arg \left(\frac{1-\gamma}{\gamma}+\ln \gamma=-\frac{c_{1}}{c_{2} \varphi(\theta)}\right) .
$$

If $-\infty<\varphi(\theta)<0$, it follows from (9) that the optimal value of the fatigue reliability index $\gamma$ exists and is unique. To make sure that the optimal solution (9) represents a minimum of (6), not a maximum, the second derivative of the performance index (6) is calculated and evaluated at $\gamma=\gamma^{*}$ :

$$
\frac{\partial^{2} C(\tau(\theta, \gamma))}{\partial \gamma^{2}}=c_{2} \varphi(\theta) \frac{1}{\gamma}\left(1-\frac{1}{\gamma}\right)>0 .
$$

which is positive (i. e., (9) represents a minimum point of (6)), as desired. Thus, the following theorem has been proven.

Theorem 1. The fatigue reliability index $\gamma$ (such that $C(\tau(\theta, \gamma))$ as defined in (6) is minimized) exists and is unique if and only if $\varphi(\theta)$ as defined in (8) satisfies the inequality: $-\infty<\varphi(\theta)<0$.

\subsection{Index of Improvement Percentage in Effectiveness of Inspection Strategy}

The index of improvement percentage in effectiveness of the optimal inspection strategy (with $\gamma=\gamma^{*}$ ) as compared with the standard inspection strategy (with $\gamma=\gamma_{\text {st }}$ ) is given by

$$
I_{\text {imp.per }}\left(\gamma^{*}, \gamma_{\mathrm{st}}\right)=\frac{C\left(\tau\left(\theta, \gamma_{\mathrm{st}}\right)-C\left(\tau\left(\theta, \gamma^{*}\right)\right.\right.}{C\left(\tau\left(\theta, \gamma_{\mathrm{st}}\right)\right.} 100 \%
$$

\subsection{Numerical Example 2.4}

Let us assume that $X$ follows the exponential distribution,

$$
\begin{gathered}
X \sim f_{\theta}(x)=(1 / \theta) \exp (-x / \theta), \\
x \geq 0, \quad \theta>0 ; \quad c_{1}=1, \quad c_{2}=15, \quad \theta=2000 .
\end{gathered}
$$

It follows from (9) and (12) that

$$
\gamma^{*}=\arg \left(\frac{1-\gamma}{\gamma}+\ln \gamma=\frac{c_{1}}{c_{2} \theta}\right)=0.991879 .
$$

It follows from (6) and (13) that

$$
\begin{aligned}
& C\left(\tau\left(\theta, \gamma^{*}\right)=\frac{c_{1}}{1-\gamma^{*}}-\frac{c_{2} \theta \ln \gamma^{*}}{1-\gamma^{*}}-c_{2} \theta\right. \\
& \quad=\frac{c_{1}}{1-\gamma^{*}}-c_{2} \theta\left(\frac{\ln \gamma^{*}}{1-\gamma^{*}}+1\right)=245.6161
\end{aligned}
$$

If the standard fatigue reliability index $\gamma_{\mathrm{st}}=0.95$ is used, then

$$
C\left(\tau\left(\theta, \gamma_{\mathrm{st}}\right)=\frac{c_{1}}{1-\gamma^{*}}-c_{2} \theta\left(\frac{\ln \gamma^{*}}{1-\gamma^{*}}+1\right)=795.9766\right.
$$

Index of improvement percentage. It follows from (11) that the index of improvement percentage in effectiveness of the optimal inspection strategy (with $\gamma=\gamma^{*}=0.991879$ ) as compared with the standard inspection strategy (with $\left.\gamma=\gamma_{\mathrm{st}}=0.95\right)$ is given by

$$
\begin{aligned}
I_{\text {imp.per }}\left(\gamma^{*}, \gamma_{\mathrm{st}}\right) & =\frac{C\left(\tau\left(\theta, \gamma_{\mathrm{st}}\right)-C\left(\tau\left(\theta, \gamma^{*}\right)\right.\right.}{C\left(\tau\left(\theta, \gamma_{\mathrm{st}}\right)\right.} 100 \% \\
& =69.1428 \% .
\end{aligned}
$$

\section{Planning in-Service Inspections Under Fatigue Crack Initiation in the Case of Parametric Uncertainty}

\subsection{Criteria for Construction of Inspection Strategies}

To construct the reliability-based inspection strategy under parametric uncertainty, the two criteria are proposed.

The first criterion, which takes into account (3) and the random sample of the past lifetime data $\left(X_{1}, \ldots, X_{n}\right)$ of the components of the same type, allows one to construct the inspection strategy given by

$$
\tau_{j}^{\text {unb }}=\arg \left(E_{\theta}\left\{\bar{F}_{\theta}(\tau(S))\right\}=\gamma^{j}\right), \quad j \geq 1,
$$

where $S$ represents some sample statistic (say, either the maximum likelihood estimator of $\theta$ or sufficient statistic for $\theta$ ). This criterion is named as 'unbiasedness criterion'.

The second criterion, which takes into account (4) and the random sample of the past lifetime data $\left(X_{1}, \ldots, X_{n}\right)$ of the components of the same type, allows one to construct the inspection strategy given by

$$
\tau_{j}^{\mathrm{mv}}=\underset{\tau(S)}{\arg \min } E_{\theta}\left\{\left[\bar{F}_{\theta}(\tau(S))-\gamma^{j}\right]^{2}\right\}, \quad j \geq 1,
$$

This criterion is named as 'minimum variance criterion'.

It will be noted that in practice, under parametric uncertainty, the criterion,

$$
\tau_{j}^{\mathrm{ml}}=\arg \left(\bar{F}_{\hat{\theta}}(\tau)=\gamma^{j}\right), \quad j \geq 1,
$$

is usually used, where $\hat{\theta}$ is the maximum likelihood estimate of $\theta$. This criterion is named as 'maximum likelihood criterion'.

When there is a fatigue reliability requirement, the problem is usually to develop an inspection strategy that meets the reliability requirements. It is assumed that only the functional form of the underlying invariant distribution of time to crack detection is specified, but some or all of its parameters are unspecified. The pivotal quantity averaging (PQA) approach proposed in this paper allows one to construct an optimal inspection strategy under parametric uncertainty.

\subsection{Inspection Strategies}

Theorem 2. Let $X_{1}, \ldots, X_{n}$ be the random sample of the past 
independent observations of time to crack detection from the fatigued structures (components) of the same type, which follow the exponential distribution with the probability density function

$$
f_{\theta}(x)=(1 / \theta) \exp (-x / \theta), \quad x \geq 0, \quad \theta>0,
$$

where the parameter $\theta$ is unknown. Then the reliability-based inspection strategies for a new fatigued component of the same type are given as follows.

The unbiased inspection strategy:

$$
\tau_{j}^{\mathrm{unb}}=\left[\gamma^{-j / n}-1\right] S, \quad j \geq 1 .
$$

The minimum variance inspection strategy:

$$
\tau_{j}^{\mathrm{mv}}=\frac{1-\gamma^{\frac{j}{n+1}}}{2 \gamma^{\frac{j}{n+1}}-1} S, j \geq 1
$$

where $S=\sum_{i=1}^{n} X_{i}$ is the sufficient statistic for $\theta$.

The maximum likelihood inspection strategy:

$$
\tau_{j}^{\mathrm{ml}}=j \ln \gamma^{-1} \frac{S}{n}, \quad j \geq 1
$$

Proof. Using the pivotal quantity averaging approach (Nechval et al. $[12,13]$ ), the unbiased inspection strategy can be obtained from (17) as

$$
\begin{gathered}
\tau_{j}^{\mathrm{unb}}=\arg \left[E_{\theta}\left\{\bar{F}_{\theta}(\tau(S))\right\}=\gamma^{j}\right] \\
=\arg \left[E_{\theta}\left\{\exp \left(-\frac{\tau_{j}}{S} \frac{S}{\theta}\right)\right\}=\gamma^{j}\right] \\
=\arg \left[E\left\{\exp \left(-\rho_{j} V\right)\right\}=\gamma^{j}\right]=\arg \left[\frac{1}{\left(1+\rho_{j}\right)^{n}}=\gamma^{j}\right] \\
=\left[\gamma^{-j / n}-1\right] S, \quad j \geq 1,
\end{gathered}
$$

where the ancillary factor $\rho_{j}$ is given by

$$
\rho_{j}=\tau_{j} / S
$$

the pivotal quantity $V$ is given by

$$
V=S / \theta \sim f(v)=\frac{1}{\Gamma(n)} v^{n-1} \exp (-v), \quad v \geq 0 .
$$

The minimum variance inspection strategy is obtained from (18) as

$$
\tau_{j}^{\mathrm{mv}}=\underset{\tau(S)}{\arg \min } E_{\theta}\left\{\left[\bar{F}_{\theta}(\tau(S))-\gamma^{j}\right]^{2}\right\}
$$

$$
\begin{gathered}
=\underset{\tau(S)}{\arg \min } E_{\theta}\left\{\left[\exp (-\tau(S) / \theta)-\gamma^{j}\right]^{2}\right\} \\
=\underset{\rho_{j}}{\arg \min } E\left\{\exp \left(-2 \rho_{j} V\right)-2 \exp \left(-\rho_{j} V\right) \gamma^{j}+\gamma^{2 j}\right\} \\
=\underset{\rho_{j}}{\arg \min _{j}}\left(\frac{1}{\left(1+2 \rho_{j}\right)^{n}}-\frac{2 \gamma^{j}}{\left(1+\rho_{j}\right)^{n}}+\gamma^{2 j}\right) \\
=\left[\left(1-\gamma^{j /(n+1)}\right) /\left(2 \gamma^{j /(n+1)}-1\right)\right] S, \quad j \geq 1 .
\end{gathered}
$$

The maximum likelihood inspection strategy follows immediately from (19):

$$
\begin{gathered}
\tau_{j}^{\mathrm{ml}}=\arg \left(\bar{F}_{\hat{\theta}}(\tau)=\gamma^{j}\right)=\arg \left[\exp \left(-\frac{\tau}{\widehat{\theta}}\right)=\gamma^{j}\right] \\
=j \ln \gamma^{-1} \frac{S}{n}, \quad j \geq 1,
\end{gathered}
$$

where the maximum likelihood estimator of $\theta$ is $\hat{\theta}=S / n$ This ends the proof.

\subsection{Optimization of Fatigue Reliability Index}

Theorem 3. If (under conditions of Theorem 2) the unbiased inspection strategy is used, where each inspection $\operatorname{costs} c_{1}$ and the cost of leaving an undetected failure (fatigue crack) is $c_{2}$ per unit time, then the fatigue reliability index $\gamma$ minimizing the total expected cost per inspection cycle is given by

$$
\gamma_{\mathrm{unb}}^{*}=\arg \min _{\gamma}\left(\frac{c_{1} n}{S} \frac{1}{1-\gamma^{(n+1) / n}}+c_{2} n \frac{\gamma^{-1 / n}-1}{1-\gamma}-c_{2}\right) .
$$

Proof. It follows from (20) and (26) that $C(\tau(\theta, \gamma))$ can be transformed as

$$
\begin{gathered}
C(\tau(\theta, \gamma))=c_{1} \sum_{j=0}^{\infty} \bar{F}_{\theta}\left(\tau_{j}\right) \\
+c_{2} \sum_{j=1}^{\infty} \tau_{j}\left[\bar{F}_{\theta}\left(\tau_{j-1}\right)-\bar{F}_{\theta}\left(\tau_{j}\right)\right]-c_{2} E_{\theta}\{X\} \\
=c_{1} \sum_{j=0}^{\infty} \exp \left(-\frac{\tau_{j}}{\theta}\right) \\
+c_{2} \sum_{j=1}^{\infty} \tau_{j}\left[\exp \left(-\frac{\tau_{j-1}}{\theta}\right)-\exp \left(-\frac{\tau_{j}}{\theta}\right)\right]-c_{2} \theta \\
=\theta\left(c_{1} \sum_{j=0}^{\infty} \frac{1}{S} V \exp \left(-\frac{\tau_{j}}{S} V\right)\right. \\
\left.+c_{2} \sum_{j=1}^{\infty} \tau_{j}\left[\frac{1}{S} V \exp \left(-\frac{\tau_{j-1}}{S} V\right)-\frac{1}{S} V \exp \left(-\frac{\tau_{j}}{S} V\right)\right]-c_{2}\right) \\
=\theta C(\tau(S, \gamma), V) .
\end{gathered}
$$


Using the pivotal quantity averaging approach, it follows from (30) that

$$
\begin{gathered}
E\{C(\tau(S, \gamma), V\} \\
=E\left\{\left(c_{1} \sum_{j=0}^{\infty} \frac{1}{S} V \exp \left(-\frac{\tau_{j}}{S} V\right)\right.\right. \\
\left.\left.+c_{2} \sum_{j=1}^{\infty} \tau_{j}\left[\frac{1}{S} V \exp \left(-\frac{\tau_{j}-1}{S} V\right)-\frac{1}{S} V \exp \left(-\frac{\tau_{j}}{S} V\right)\right]-c_{2}\right)\right\} \\
=\frac{c_{1}}{S} \sum_{j=0}^{\infty} n\left(1+\frac{\tau_{j}}{S}\right)^{-1} c^{-1} \sum_{j=1}^{\infty} \tau_{j}\left[n\left(1+\frac{\tau_{j-1}}{S}\right)^{-1}-n\left(1+\frac{\tau_{j}}{S}\right)^{-1}\right]-c_{2} \\
=C(\tau(S, \gamma)) .
\end{gathered}
$$

It follows from (24) and (31) that

$$
\begin{gathered}
C\left(\tau^{\mathrm{unb}}(S, \gamma)\right)=\frac{c_{1}}{S} \sum_{j=0}^{\infty} n\left(1+\frac{\tau_{j}^{\mathrm{unb}}}{S}\right)^{-1} \\
+\frac{c_{2}}{S} \sum_{j=1}^{\infty} \tau_{j}^{\mathrm{unb}}\left[n\left(1+\frac{\tau_{j-1}^{\mathrm{unb}}}{S}\right)^{-1}-n\left(1+\frac{\tau_{j}^{\mathrm{unb}}}{S}\right)^{-1}\right]-c_{2} \\
\left.=\frac{c_{1} n}{S} \sum_{j=0}^{\infty} \gamma^{j(n+1) / n}\right]-c_{2}^{n} \sum_{j=1}^{\infty}\left(\gamma^{-j / n}-1\right)\left[\gamma^{(j-1)(n+1) / n}-\gamma^{j(n+1) / n}\right]-c_{2} \\
=\frac{c_{1} n}{S} \frac{1}{1-\gamma^{(n+1) / n}}+c_{2} n \sum_{j=1}^{\infty}\left(\gamma^{-j / n}-1\right)\left[\gamma^{(j-1) / n}-\gamma^{j / n}\right]-c_{2} \\
=\frac{c_{1} n}{S} \frac{1}{1-\gamma^{(n+1) / n}} \\
+c_{2} n \sum_{j=1}^{\infty}\left(\gamma^{j-1} \gamma^{-1 / n}-\gamma^{(j-1)(n+1) / n}\right)\left[1-\gamma^{(n+1) / n}\right]-c_{2} \\
=\frac{c_{1} n}{S} \frac{1}{1-\gamma^{(n+1) / n}}+c_{2} n \frac{\gamma^{-1 / n}-1}{1-\gamma}-c_{2} \cdot{ }^{(32)}
\end{gathered}
$$

Thus, we can choose the fatigue reliability index $\gamma$ such that $C\left(\tau^{\mathrm{unb}}(S, \gamma)\right)$ as defined in (32) is minimized. The optimal value of the fatigue reliability index $\gamma$ is determined as

$$
\begin{gathered}
\gamma_{\mathrm{unb}}^{*}=\underset{\gamma}{\arg \min C\left(\tau^{\mathrm{unb}}(S, \gamma)\right)} \\
=\arg \min _{\gamma}\left(\frac{c_{1} n}{S} \frac{1}{1-\gamma^{(n+1) / n}}+c_{2} n \frac{\gamma^{-1 / n}-1}{1-\gamma}-c_{2}\right) .
\end{gathered}
$$

This ends the proof.

\subsection{Index of Improvement Percentage in Effectiveness of Inspection Strategy}

The index of improvement percentage in effectiveness of the optimal unbiased inspection strategy (with $\gamma=\gamma_{\text {unb }}^{*}$ ) as compared with the standard inspection strategy (with $\left.\gamma=\gamma_{\text {st }}\right)$ is given by

$$
I_{\text {imp.per }}\left(\gamma_{\mathrm{unb}}^{*}, \gamma_{\mathrm{st}}\right)=\frac{C\left(\tau\left(S, \gamma_{\mathrm{st}}\right)-C\left(\tau\left(S, \gamma_{\mathrm{unb}}^{*}\right)\right.\right.}{C\left(\tau\left(S, \gamma_{\mathrm{st}}\right)\right.} 100 \% \text {. }
$$

\subsection{Numerical Example 3.5}

Let $X_{1}, \ldots, X_{n}$ be the random sample of the past independent observations of time to crack detection (from the same fatigued structures) which follow the exponential distribution (20), where $n=2$ and the parameter $\theta$ is unknown. The sufficient statistic for $\theta$ is $S=4000$ hours. In order to construct the reliability-based inspection strategy for a new fatigued structure of the same type, the unbiased inspection strategy (21) will be used. Let us assume that each inspection costs $c_{1}=1$ (in terms of money) and the cost of leaving an undetected failure (fatigue crack) is $c_{2}=15$ (in terms of money) per unit time. Then it follows from (33) that

$$
\begin{gathered}
\gamma_{\mathrm{unb}}^{*}==\arg \min _{\gamma}\left(\frac{c_{1} n}{S} \frac{1}{1-\gamma^{(n+1) / n}}+c_{2} n \frac{\gamma^{-1 / n}-1}{1-\gamma}-c_{2}\right) \\
=0.994581 .
\end{gathered}
$$

It follows from (32) and (35) that

$$
\begin{gathered}
C\left(\tau^{\mathrm{unb}}\left(S, \gamma_{\mathrm{unb}}^{*}\right)=\frac{c_{1} n}{S} \frac{1}{1-\left(\gamma_{\mathrm{unb}}^{*}\right)^{(n+1) / n}}\right. \\
+c_{2} n \frac{\left(\gamma_{\mathrm{unb}}^{*}\right)^{-1 / n}-1}{1-\gamma_{\mathrm{unb}}^{*}}-c_{2}=0.122836 .
\end{gathered}
$$

If the standard fatigue reliability index $\gamma_{\mathrm{st}}=0.95$ is used, then

$$
\begin{aligned}
& C\left(\tau^{\mathrm{unb}}\left(S, \gamma_{\mathrm{st}}\right)=\frac{c_{1} n}{S} \frac{1}{1-\left(\gamma_{\mathrm{st}}\right)^{(n+1) / n}}\right. \\
& +c_{2} n \frac{\left(\gamma_{\mathrm{st}}\right)^{-1 / n}-1}{1-\gamma_{\mathrm{st}}}-c_{2}=0.593763
\end{aligned}
$$

Index of improvement percentage. It follows from (34) that the index of improvement percentage in effectiveness of the optimal unbiased inspection strategy (with $\left.\gamma=\gamma_{\text {unb }}^{*}=0.994581\right)$ as compared with the standard unbiased inspection strategy (with $\gamma=\gamma_{\mathrm{st}}=0.95$ ) is given by 


$$
\begin{aligned}
I_{\text {imp.per }}\left(\gamma_{\mathrm{unb}}^{*}, \gamma_{\mathrm{st}}\right) & =\frac{C\left(\tau\left(S, \gamma_{\mathrm{st}}\right)-C\left(\tau\left(S, \gamma_{\mathrm{unb}}^{*}\right)\right.\right.}{C\left(\tau\left(S, \gamma_{\mathrm{st}}\right)\right.} 100 \% \\
& =79.3123 \%
\end{aligned}
$$

\subsection{Numerical Example 3.6}

Consider, for example, the data of fatigue tests on a particular type of structural components (stringer) of aircraft IL-86. The data are for a complete sample of size $n=5$, with observations of time to crack initiation (in number of $10^{4}$ flight hours): $X_{1}=5, X_{2}=6.25, X_{3}=7.5, X_{4}=7.9, X_{5}=8.1$.

Goodness-of-fit Testing. It is assumed that $X_{i}, i=1(1) 5$, follow the two-parameter Weibull distribution with the probability distribution function

$$
\operatorname{Pr}\{X \leq x\}=1-\exp \left[-\left(\frac{x}{\beta}\right)^{\delta}\right], \mathrm{x} \geq 0,
$$

where the parameters $\beta$ and $\delta$ are unknown. We assess the statistical significance of departures from the Weibull model by performing empirical distribution function goodness-of-fit test. The $K$ statistic (Kapur and Lamberson [14]) is used. For censoring (or complete) datasets, the $K$ statistic is given by

$$
K=\frac{\sum_{i=[r / 2]+1}^{r-1}\left(\frac{\ln \left(x_{i+1} / x_{i}\right)}{M_{i}}\right)}{\sum_{i=1}^{r-1}\left(\frac{\ln \left(x_{i+1} / x_{i}\right)}{M_{i}}\right)}=\frac{\sum_{i=3}^{4}\left(\frac{\ln \left(x_{i+1} / x_{i}\right)}{M_{i}}\right)}{\sum_{i=1}^{4}\left(\frac{\ln \left(x_{i+1} / x_{i}\right)}{M_{i}}\right)}=0.184,
$$

where $[r / 2]$ is a largest integer $\leq r / 2$, the values of $M_{i}$ are given in [14]. The rejection region for the $\alpha$ level of significance is $\left\{K>K_{n ; \alpha}\right\}$. The percentage points for $K_{n ; \alpha}$ were given by Kapur and Lamberson [14]. For this example, where $r=n=5$,

$$
K=0.184<K_{n=5 ; \alpha=0.05}=0.86 \text {. }
$$

Thus, there is not evidence to rule out the Weibull model.

Theorem 4. Let $X_{1} \leq \ldots \leq X_{r}$ be the first $r$ ordered past observations of time to crack detection from the $n$ fatigued structures of the same type, which follow the Weibull distribution with the probability distribution function (39)

$$
\operatorname{Pr}\{X \leq x\}=1-\exp \left[-\left(\frac{x}{\beta}\right)^{\delta}\right], x \geq 0 .
$$

Then the unbiased reliability-based strategy of inspections of a new fatigued structure of the same type is given as follows:

$$
\tau_{j}=\arg \left[\operatorname{Pr}\left\{Y_{1}>\tau_{j} \mid \mathbf{w}\right\}=\gamma^{j}\right]=\arg \left[\frac{\int_{0}^{\infty} v_{2}^{r-2} \prod_{i=1}^{r} w_{i}^{v_{2}}\left(w_{\tau_{j}}^{v_{2}}+\sum_{i=1}^{r} w_{i}^{v_{2}}+(n-r) w_{r}^{v_{2}}\right)^{-r} d v_{2}}{\int_{0}^{\infty} v_{2}^{r-2} \prod_{i=1}^{r} w_{i}^{v_{2}}\left(\sum_{i=1}^{r} w_{i}^{v_{2}}+(n-r) w_{r}^{v_{2}}\right)^{-r} d v_{2}}=\gamma^{j}\right], \mathrm{j} \geq 1,
$$

where

$$
\begin{gathered}
V_{2}=\frac{\delta}{\widehat{\delta}}, \quad \mathbf{w}=\left(w_{1}, w_{2}, \ldots, w_{r}\right), \\
W_{i}=\left(\frac{X_{i}}{\widehat{\beta}}\right)^{\widehat{\delta}}, \quad i=1, \ldots, r, \quad w_{\tau_{j}}=\left(\frac{\tau_{j}}{\widehat{\beta}}\right)^{\widehat{\delta}},
\end{gathered}
$$

$\widehat{\beta}$ and $\widehat{\delta}$ are the maximum likelihood estimators of $\beta$ and $\delta$ based on the first $r$ ordered past observations $(\mathrm{X} 1 \leq \ldots \leq \mathrm{Xr})$ from a sample of size $\mathrm{n}$ from the two-parameter Weibull distribution (39), which can be found from solution of

$$
\widehat{\beta}=\left(\left[\sum_{i=1}^{r} x_{i}^{\widehat{\delta}}+(n-r) x_{r}^{\widehat{\delta}}\right] / r\right)^{1 / \widehat{\delta}},
$$

and

\begin{tabular}{|c|c|c|c|c|c|c|c|}
\hline \multicolumn{8}{|c|}{ Inspection time (in terms of flight hours) $\tau_{j, j}=0(1) 9$} \\
\hline$\tau_{0}$ & $\tau_{1}$ & $\tau_{2}$ & $\tau_{3}$ & $\tau_{4}$ & $\tau_{5}$ & $\tau_{6}$ & $\tau_{7}$ \\
\hline 0 & 25549 & 32569 & 36975 & 40212 & 42775 & 44898 & 46708 \\
\hline$\tau_{8}$ & $\tau_{9}$ & ... & ... & $\ldots$ & $\ldots$ & $\ldots$ & $\ldots$ \\
\hline 48287 & 49685 & $\ldots$ & $\ldots$ & $\ldots$ & $\ldots$ & $\ldots$ & $\ldots$ \\
\hline \multicolumn{8}{|c|}{ Interval (in terms of flight hours) $\tau_{j+1}-\tau_{j, j}=0(1) 8$} \\
\hline- & $\tau_{1}-\tau_{0}$ & $\tau_{2}-\tau_{1}$ & $\tau_{3}-\tau_{2}$ & $\tau_{4}-\tau_{3}$ & $\tau_{5}-\tau_{4}$ & $\tau_{6}-\tau_{5}$ & $\tau_{7}-\tau_{6}$ \\
\hline- & 25549 & 7020 & 4406 & 3237 & 2563 & 2123 & 1810 \\
\hline$\tau_{8}-\tau_{7}$ & $\tau_{9}-\tau_{8}$ & $\ldots$ & $\ldots$ & $\ldots$ & $\ldots$ & $\ldots$ & $\ldots$ \\
\hline 1579 & 1398 & $\ldots$ & $\ldots$ & $\ldots$ & $\ldots$ & $\ldots$ & $\ldots$ \\
\hline
\end{tabular}

$$
\widehat{\delta}=\left[\begin{array}{l}
\left(\sum_{i=1}^{r} x_{i}^{\widehat{\delta}} \ln x_{i}+(n-r) x_{r}^{\widehat{\delta}} \ln x_{r}\right) \\
\times\left(\sum_{i=1}^{r} x_{i}^{\widehat{\delta}}+(n-r) x_{r}^{\hat{\delta}}\right)^{-1}-\frac{1}{r} \sum_{i=1}^{r} \ln x_{i}
\end{array}\right]^{-1} .
$$

Proof. The proof is similar to that of Theorem 2 and so it is omitted here.

Inspection planning under fatigue crack initiation. Using (43), for $\gamma=0.95$, we have obtained the following inspection time sequence (see Table 1). Graphical representation of inspection intervals is shown in Figure 2.

Table 1. Inspection time sequence under fatigue crack initiation. 


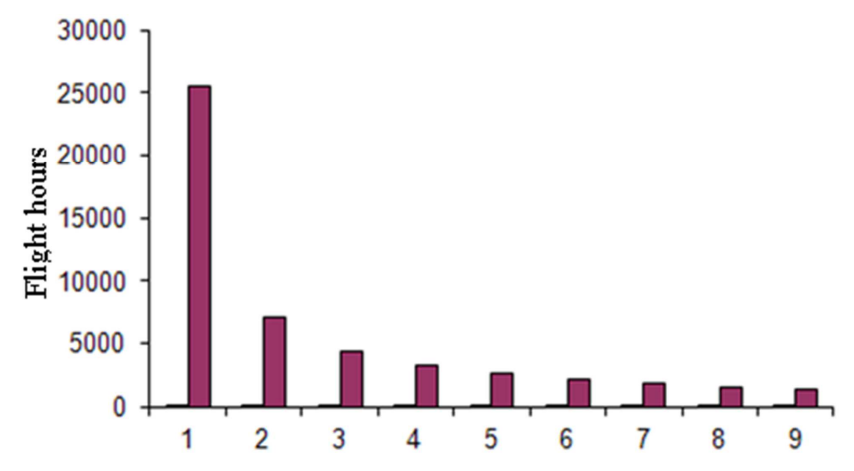

Figure 2. Graphical representation of inspection intervals under fatigue crack initiation.

\section{Planning in-Service Inspections Under Fatigue Crack Propagation (Growth)}

To capture the statistical nature of fatigue crack growth, different stochastic models have been proposed in the literature. Some of the models are purely based on direct curve fitting of the random crack growth data, including their mean value and standard deviation (Bogdanoff and Kozin [15]). These models, however, have been criticized by other researchers, because less crack growth mechanisms have been included in them. To overcome this difficulty, many probabilistic models adopted the crack growth equations proposed by fatigue experimentalists, and randomized the equations by including random factors into them (Lin and Yang [16]; Yang et al. [17]; Yang and Manning [18]; Nechval et al. [19, 20]; Straub and Faber [21]). The random factor may be a random variable, a random process of time, or a random process of space. It then creates a random differential equation. The solution of the differential equation reveals the probabilistic nature as well as the scatter phenomenon of the fatigue crack growth. To justify the applicability of the probabilistic models mentioned above, fatigue crack growth data are needed. However, it is rather time-consuming to carry out experiments to obtain a set of statistical meaningful fatigue crack growth data. To the writers' knowledge, there are only a few data sets available so far for researchers to verify their probabilistic models. Among them, the most famous data set perhaps is the one produced by Virkler et al. [22]. More frequently used data sets include one reported by Ghonem and Dore [23]. Itagaki and his associates have also produced some statistically meaningful fatigue crack growth data, but have not been mentioned very often (Itagaki et al. [24]). In fact, many probabilistic fatigue crack growth models are either lack of experimental verification or just verified by only one of the above data sets. It is suspected that a model may explain a data set well but fail to explain another data set. The universal applicability of many probabilistic models still needs to be checked carefully by other available data sets.

Many probabilistic models of fatigue crack growth are based on the deterministic crack growth equations. The most well known equation is

$$
\frac{d a(t)}{d t}=q(a(t))^{b}
$$

in which $q$ and $b$ are constants to be evaluated from the crack growth observations. The independent variable $t$ can be interpreted as stress cycles, flight hours, or flights depending on the applications. It is noted that the power-law form of $q(a(t))^{b}$ at the right hand side of (47) can be used to fit some fatigue crack growth data appropriately and is also compatible with the concept of Paris-Erdogan law (Paris and Erdogan [25]).

\subsection{Innovative Stochastic Model of Fatigue Crack Propagation}

This model of fatigue crack propagation (growth) is based on the following deterministic crack growth equation,

$$
\frac{d t(a)}{d a}=\frac{q_{1} q_{0}}{a^{2}} \exp \left(-\frac{q_{0}}{a}\right),
$$

in which $q_{1}$ and $q_{0}$ are constants to be evaluated from the crack growth observations. The variable $t$ can be interpreted as stress cycles, flight hours, or flights depending on the applications. The service time for a crack to grow from size $a_{0}$ to $a$ (where $a>$ $a_{0}$ ) can be found by performing the necessary integration

$$
\int_{t\left(a_{0}\right)}^{t(a)} d t=q_{1} \int_{a_{0}}^{a} \frac{q_{0}}{v^{2}} \exp \left(-\frac{q_{0}}{v}\right) d v
$$

to obtain

$$
t(a)-t\left(a_{0}\right)=q_{1}\left[\exp \left(-\frac{q_{0}}{a}\right)-\exp \left(-\frac{q_{0}}{a_{0}}\right)\right] .
$$

If $a_{0}=0$, then it follows from (50) that

$$
t(a)=q_{1} \exp \left(-\frac{q_{0}}{a}\right)
$$

or

$$
a \ln t(a)=\beta_{0}+\beta_{1} a,
$$

where $\beta_{1}=\ln q_{1}, \quad \beta_{0}=-q_{0}$. Including a stochastic factor $\varepsilon$ (say, $\varepsilon \sim \mathrm{N}(0, \sigma 2)$ ) into (52), we obtain the innovative stochastic model of fatigue crack propagation

$$
a \ln t(a)=\beta_{0}+\beta_{1} a+\varepsilon .
$$

\subsection{New Equation for Planning in-Service Inspections Under Fatigue Crack Propagation}

Let us assume that we have a sample of $k$ data points consisting of pairs of observed values of $a$ and $\tau$, say $\left(\tau_{1}, a_{1}\right)$, $\left(\tau_{2}, a_{2}\right), \ldots,\left(\tau_{k}, a_{k}\right)$, where $k>2, \tau_{j}$ is the time of the $j$ th inspection, $a_{j}$ is the crack size detected by means of the $j$ th 
inspection, $j=1(1) k$. Then, taking into account (53), it can be shown that the time $\tau_{k+1}$ of the next inspection is determined as

$$
\tau_{k+1}=\exp \left(\frac{h_{k+1}^{(1-\alpha)}}{\tilde{a}_{k+1}}\right)
$$

where

$$
\tilde{a}_{k+1}=a_{k}+\Delta_{k}
$$

represents an assumed value of future crack size $a_{k+1}$ (not yet observed), $\Delta_{k}=\tilde{a}_{k+1}-a_{k}$ represents an increment of $a_{k}$,

$$
\begin{gathered}
h_{k+1}^{(1-\alpha)}=\widehat{\beta}_{0}+\widehat{\beta}_{1} \tilde{a}_{k+1} \\
-t_{k-2 ; 1-\alpha} \hat{\sigma}\left[\begin{array}{c}
\left(\tilde{a}_{k+1}-\frac{1}{k} \sum_{j=1}^{k} a_{j}\right)^{2} \\
(k-1)\left[\sum_{j=1}^{k} a_{j}^{2}-\frac{1}{k}\left(\sum_{j=1}^{k} a_{j}\right)^{2}\right]
\end{array}\right]
\end{gathered}
$$

represents the $100(1-\alpha) \%$ lower prediction limit for a single future value of $\mathrm{ak}+1 \ln \tau \mathrm{k}+1, \quad t_{n-2 ; 1-\alpha}$ denotes the $(1-\alpha)$ quantile of the t-distribution with $(n-2)$ degrees of freedom,

$$
\begin{gathered}
\widehat{\beta}_{0}=\frac{1}{k} \sum_{j=1}^{k} a_{j} \ln \tau_{j}-\widehat{\beta}_{1} \frac{1}{k} \sum_{j=1}^{k} a_{j}, \\
\widehat{\beta}_{1}=\frac{\sum_{j=1}^{k} a_{j}^{2} \ln \tau_{j}-\frac{1}{k}\left(\sum_{j=1}^{k} a_{j}\right)\left(\sum_{j=1}^{k} a_{j} \ln \tau_{j}\right)}{\sum_{j=1}^{k} a_{j}^{2}-\frac{1}{k}\left(\sum_{j=1}^{k} a_{j}\right)^{2}} \\
\hat{\sigma}=\left[\frac{1}{k-2} \sum_{j=1}^{k}\left(a_{j} \ln \tau_{j}-\widehat{\beta}_{0}-\widehat{\beta}_{1} a_{j}\right)^{2}\right]^{1 / 2} .
\end{gathered}
$$

\subsection{Numerical Example 4.3}

For illustration, the procedure of inspection planning based on the innovative model (53) was used for the upper longeron of RNLAF F-16 aircraft [26] (Figure 3).
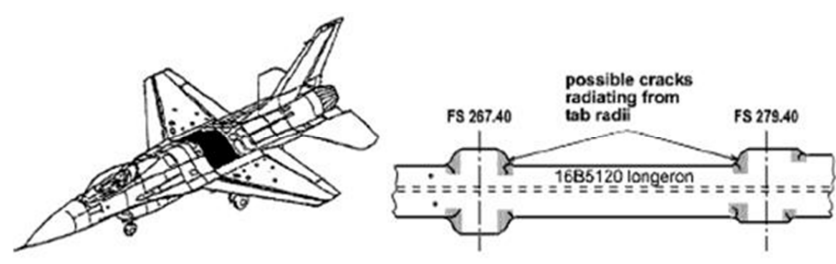

Figure 3. Inspection points of the upper longeron of RNLAF F-16 aircraft.
Figure 4 shows the deterministic damage tolerance inspection requirements (Military Specification [26]) for the RNLAF longerons.

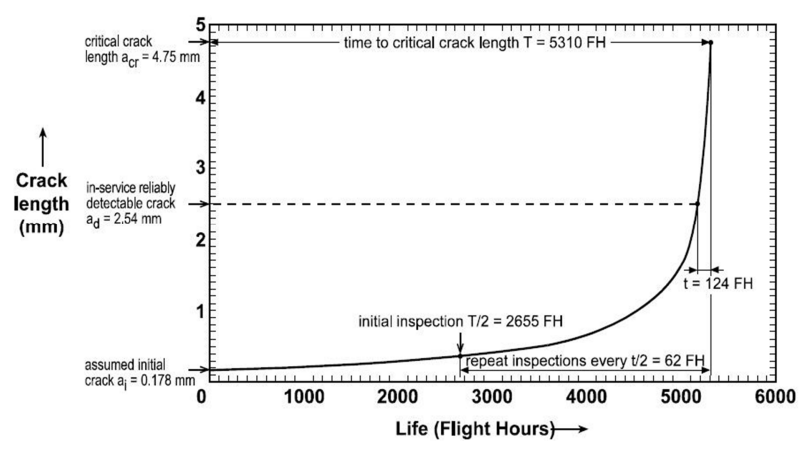

Figure 4. Deterministic damage tolerance inspection requirements for the $R N L A F$ longerons.

These requirements led to the following inspection scheme which includes $N_{\text {const.int. }}^{\text {inspections. }}=42$ inspections: (i) initial inspection after 2655 flights hours; (ii) repeat inspections every 62 flight hours. It will be noted that this inspection scheme has an unknown safety level.

Using the RNLAF longeron mean crack growth curve (Figure 4) and the innovative stochastic model of fatigue crack propagation (53), we have obtained from (54), where the safety level was specified to be $1-\alpha=0.99, \Delta_{k}=4 \mathrm{~mm}$ for all $k$, the following in-service inspection time sequence with decreasing intervals (see Table 2).

Table 2. Inspection time sequence under fatigue crack propagation.

\begin{tabular}{llllllll}
\hline \multicolumn{7}{l}{ Inspection time (in terms of flight hours) } & $\tau_{j, j} \mathbf{j}=\mathbf{1}(\mathbf{1}) \mathbf{1 2}$ \\
\hline$\tau_{1}$ & $\tau_{2}$ & $\tau_{3}$ & $\tau_{4}$ & $\tau_{5}$ & $\tau_{6}$ & $\tau_{7}$ & $\tau_{8}$ \\
\hline 2655 & 4200 & 4600 & 4800 & 4930 & 5030 & 5110 & 5170 \\
$\tau_{9}$ & $\tau_{10}$ & $\tau_{11}$ & $\tau_{12}$ & $\ldots$ & $\ldots$ & $\ldots$ & $\ldots$ \\
5220 & 5260 & 5290 & 5310 & $\ldots$ & $\ldots$ & $\ldots$ & $\ldots$ \\
Interval (in terms & of flight hours) & $\tau_{j+1}-\tau_{j, j}=0(1) 11, \tau_{0}=0$ & & \\
$\tau_{1}-\tau_{0}$ & $\tau_{2}-\tau_{1}$ & $\tau_{3}-\tau_{2}$ & $\tau_{4}-\tau_{3}$ & $\tau_{5}-\tau_{4}$ & $\tau_{6}-\tau_{5}$ & $\tau_{7}-\tau_{6}$ & $\tau_{8}-\tau_{7}$ \\
2655 & 1545 & 400 & 200 & 130 & 100 & 80 & 60 \\
$\tau_{5}-\tau_{8}$ & $\tau_{10}-\tau_{9}$ & $\tau_{11}-\tau_{10}$ & $\tau_{12}-\tau_{11}$ & $\ldots$ & $\ldots$ & $\ldots$ & $\ldots$ \\
50 & 40 & 30 & 20 & $\ldots$ & $\ldots$ & $\ldots$ & $\ldots$ \\
\hline
\end{tabular}

Graphical representation of decreasing in-service inspection intervals under crack propagation for the proposed inspection scheme (Table 2) is shown in Figure 5. Thus, the proposed inspection scheme contains only $N_{\text {decr.int. }}^{\text {inspections }}=12$ inspections.

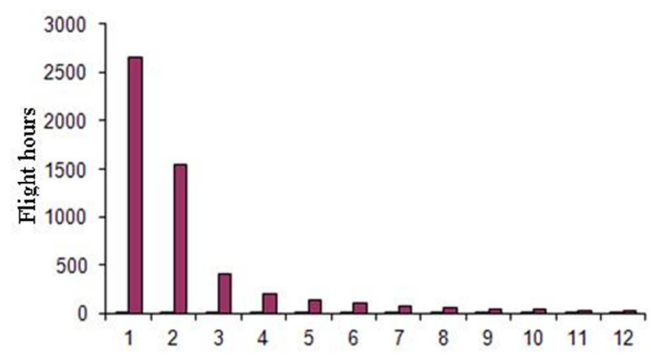

Figure 5. Graphical representation of inspection intervals under fatigue crack propagation. 
The index of relative efficiency of the inspection scheme with constant intervals as compared with the inspection scheme with decreasing intervals is given by

$$
\begin{gathered}
I_{\text {rel.eff. }}\left(N_{\text {const.int. }}^{\text {inspections }}, N_{\text {decr.int. }}^{\text {inspections }}\right) \\
=N_{\text {decr.int. }}^{\text {inspections }} / N_{\text {const.int. }}^{\text {inspections }}=12 / 42=0.29 .
\end{gathered}
$$

The index of reduction percentage in the inspection costs for the inspection scheme with decreasing intervals as compared with the inspection scheme with constant intervals is given by

$$
\begin{gathered}
I_{\text {red.per. }}\left(N_{\text {decr.int. }}^{\text {inspections }}, N_{\text {const.int. }}^{\text {inspections }}\right) \\
=\left[1-I_{\text {rel.eff. }}\left(N_{\text {const.int. }}^{\text {inspections }}, N_{\text {decr.int. }}^{\text {inspections }}\right)\right] 100 \%=71 \% .
\end{gathered}
$$

\section{Conclusion}

The technique proposed in this paper represents a simple and computationally attractive statistical method based on the constructive use of the invariance principle in mathematical statistics. The main advantage of this technique consists in that it allows one to eliminate unknown parameters from the problem and to use the past lifetime data for planning future inspections as completely as possible. The unbiasedness and minimum variance criteria, which are proposed in the paper for constructing inspection strategies under fatigue crack initiation such as the unbiased inspection strategy (UIS) and minimum variance inspection strategy (MVIS), respectively, represent the novelty of the work. It is clear that these inspection strategies, which have such properties as unbiasedness and minimum variance, are preferable as compared to the maximum likelihood inspection strategy (MLIS). We have illustrated the prediction methods for log-location-scale distributions (such as the Exponential, Gumbel or Weibull distribution). Application to other distributions could follow directly. Under fatigue crack growth, the damage tolerance situation is considered. As a result of our investigations of the experimental data of fatigue crack growth, we have found that for planning in-service inspections of fatigued structures under crack propagation it can be used the approach based on a novel crack growth equation to construct more accurate reliability-based inspection strategy in this case. The new technique proposed for planning in-service inspections of fatigued structures under crack propagation requires a quantile of the $t$-distribution and is conceptually simple and easy to use. The results obtained in this work can be used to solve the service problems of the following important engineering structures: (1) Transportation Systems and Vehicles - aircraft, space vehicles, trains, ships; (2) Civil Structures - bridges, dams, tunnels; (3) Power Generation - nuclear, fossil fuel and hydroelectric plants; (4) High-Value Manufactured Products - launch systems, satellites, semiconductor and electronic equipment; (5) Industrial Equipment - oil and gas exploration, production and processing equipment, chemical process facilities, pulp and paper.

\section{References}

[1] N. Iyyer, S. Sarkar, R. Merrill, and N. Phan, "Aircraft life management using crack initiation and crack growth models P-3C Aircraft experience," International Journal of Fatigue, vol. 29, pp. 1584-1607, 2007.

[2] R. E. Barlow, L. C. Hunter, and F. Proschan, "Optimum checking procedures," Journal of the Society for Industrial and Applied Mathematics, vol. 11, 1078-1095, 1963.

[3] H. Luss and Z. Kander, "Inspection policies when duration of checkings is non-negligible," Operational Research Quarterly, vol. 25, pp. 299-309, 1974.

[4] B. Sengupta, "Inspection procedures when failure symptoms are delayed," Operational Research Quarterly, vol. 28, pp. 768-776, 1977.

[5] A. G. Munford and A. K. Shahani, "A nearly optimal inspection policy,” Operational Research Quarterly, vol. 23, pp. 373-379, 1972.

[6] A. G. Munford and A. K. Shahani, (1973). "An inspection policy for the Weibull case," Operational Research Quarterly, vol. 24, pp. 453-458, 1973.

[7] P. R. Tadikamalla, "An inspection policy for the gamma failure distributions," Operational Research Quarterly, vol. 30, pp. 77-78, 1979.

[8] A. G. Munford, "Comparison among certain inspection policies," Management Science, vol. 27, pp. 260-267, 1981.

[9] N. A. Nechval, K. N. Nechval, G. Berzins, M. Purgailis, and U. Rozevskis, "Stochastic fatigue models for efficient planning inspections in service of aircraft structures," in K. Al-Begain, A. Heindl, and M. Telek (Eds.), Analytical and Stochastic Modeling Techniques and Applications, Lecture Notes in Computer Science (LNCS), vol. 5055.. Berlin, Heidelberg: Springer-Verlag, 2008, pp. 114-127.

[10] K. N. Nechval, N. A. Nechval, G. Berzins, M. Purgailis, U. Rozevskis, and V. F. Strelchonok, "Optimal adaptive inspection planning process in service of fatigued aircraft structures," in K Al-Begain, D. Fiems, and G. Horvath (Eds.), Analytical and Stochastic Modeling Techniques and Applications, Lecture Notes in Computer Science (LNCS), vol. 5513. Berlin, Heidelberg: Springer-Verlag, 2009, pp. 354-369.

[11] N. A. Nechval, K. N. Nechval, and M. Purgailis, "Inspection policies in service of fatigued aircraft structures," in S. I. Ao and L. Gelman (Eds.), Electrical Engineering and Applied Computing, Lecture Notes in Electrical Engineering, vol. 90. Berlin, Heidelberg: Springer Science+Business Media B. V., 2011, pp. 459-472.

[12] N. A. Nechval, G. Berzins, M. Purgailis, and K. N. Nechval, "Improved estimation of state of stochastic systems via invariant embedding technique," WSEAS Transactions on Mathematics, vol. 7, pp. 141-159, 2008.

[13] N. A. Nechval, K. N. Nechval, and M. Purgailis, "Prediction of future values of random quantities based on previously observed data," Engineering Letters, vol. 9, pp. 346-359, 2011.

[14] K. C. Kapur and L. R. Lamberson, Reliability in Engineering Design. New York: Wiley, 1977. 
[15] J. L. Bogdanoff and F. Kozin, Probabilistic Models of Cumulative Damage. New York: Wiley, 1985.

[16] Y. K. Lin and J. N. Yang, "On statistical moments of fatigue crack propagation," Engineering Fracture Mechanics, vol. 18, 243-256, 1985.

[17] J. N. Yang, W. H. His, and S. D. Manning, "Stochastic crack propagation with applications to durability and damage tolerance analyses," Technical Report, Flight Dynamics Laboratory, Wright-Patterson Air Force Base, AFWAL-TR-85-3062, 1985.

[18] J. N. Yang and S. D. Manning, "Stochastic crack growth analysis methodologies for metallic structures," Engineering Fracture Mechanics, vol. 37, pp. 1105-1124, 1990.

[19] N. A. Nechval, K. N. Nechval, and E. K. Vasermanis, "Statistical models for prediction of the fatigue crack growth in aircraft service," in A. Varvani-Farahani and C. A. Brebbia (Eds.), Fatigue Damage of Materials 2003. Southampton, Boston: WIT Press, 2003, pp. 435-445.

[20] N. A. Nechval, K. N. Nechval, and E. K. Vasermanis, "Estimation of warranty period for structural components of aircraft," Aviation, vol. VIII, pp. 3-9, 2004.
[21] D. Straub and M. H. Faber, "Risk based inspection planning for structural systems," Structural Safety, vol. 27, pp. 335-355, 2005.

[22] D. A. Virkler, B. M. Hillberry, and P. K. Goel, "The statistic nature of fatigue crack propagation," ASME Journal of Engineering Materials and Technology, vol. 101, pp. 148-153, 1979.

[23] H. Ghonem and S. Dore, "Experimental study of the constant probability crack growth curves under constant amplitude loading,” Engineering Fracture Mechanics, vol. 27, pp. 1-25, 1987.

[24] H. Itagaki, T. Ishizuka, and P. Y. Huang, "Experimental estimation of the probability distribution of fatigue crack growth lives," Probabilistic Engineering Mechanics, vol. 8, pp. 25-34, 1993.

[25] R. Paris and F. Erdogan, "A critical analysis of crack propagation laws," Journal of Basic Engineering, vol. 85, pp. 528-534, 1963.

[26] Military Specification, Airplane Damage Tolerance Requirements. MIL-A-83444 (USAF), 1974. 